\title{
El gusano cogollero del maíz Spodoptera frugiperda (J.E. Smith) (Lepidoptera: Noctuidae) y algunas plantas transgénicas
}

\author{
The fall armyworm Spodoptera frugiperda (J.E. Smith) \\ (Lepidoptera: Noctuidae) and some transgenic plants
}

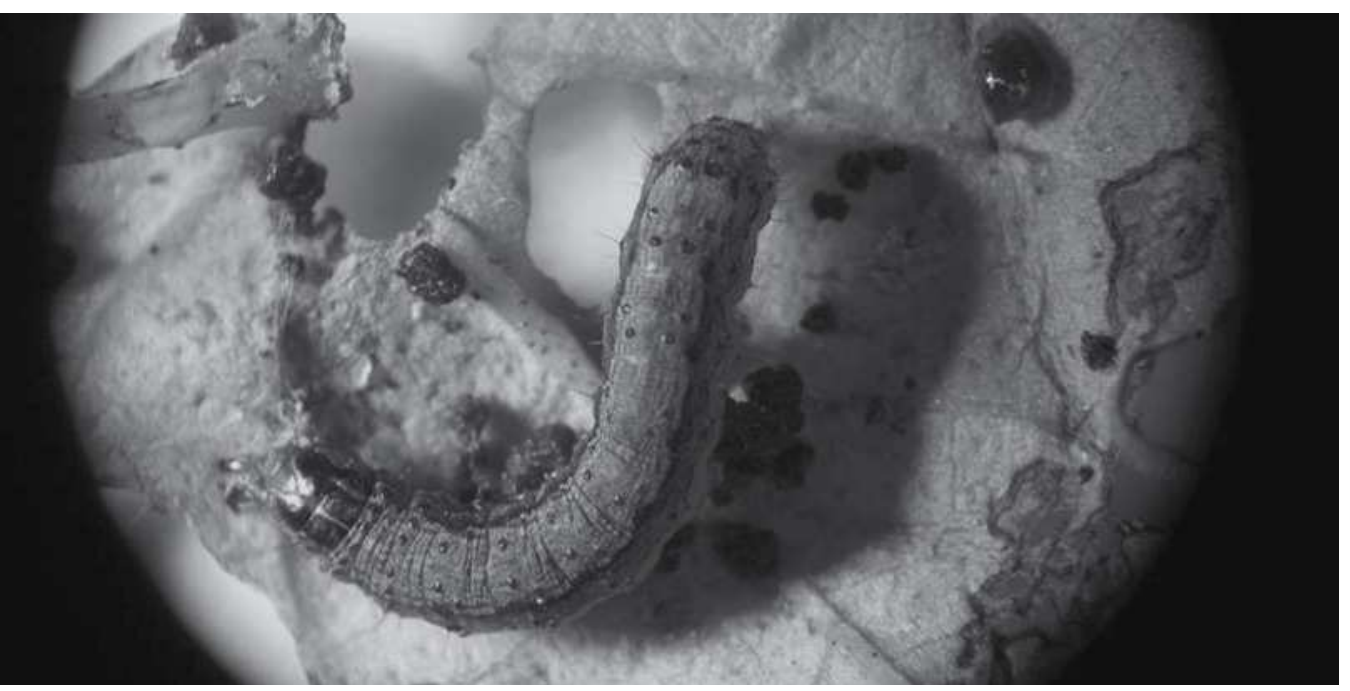

INGEBORG ZENNER DE POLANÍA'

HELBER A. ARÉVALO2

RODOLFO MEJÍA ${ }^{3}$

IV instar del cogollero del maíz,

alimentándose de algodón

transgénico.

Foto: I. Zenner de Polanía

\section{RESUMEN}

Basado en datos experimentales preliminares y una crítica revisión de estudios realizados en Norteamérica y Suramérica, se analiza y se discute la posibilidad de la existencia en Colombia de dos razas o biotipos del cogollero del maíz, Spodoptera frugiperda, su influencia sobre el manejo de esta plaga polífaga y las perspectivas de su control con variedades o híbridos de maíz y algodonero transgénico que expresan una o más toxinas del Bacillus thuringiensis. Se propone, fundamentado en la similitud de las $\mathrm{CL}_{50}$ determinadas a través de evaluaciones por tolerancia a la toxina Cry1Ac del Bt de diversas razas del cogollero, recolectados en diferentes huéspedes y regiones geográficas, la existencia de la raza que ataca tanto maíz como al algodonero y la raza que afecta arroz y pastos. Poblaciones procedentes de maíz y algodón mostraron una tolerancia hasta 127 veces mayor que la población colectada en pastos. Esta última raza exhibió además una $\mathrm{CL}_{50}$ entre 3,45 y 5,64 ppm al Cry1 Ab. Igualmente, se muestran y evalúan algunos avances a nivel internacional en la obtención de especies vegetales, huéspedes del cogollero, genéticamente transformadas, con toxinas diferentes a las procedentes del Bt, huéspedes del cogollero.

Docente Investigadora, Facultad de Ingeniería Agronómica, Universidad de Ciencias Aplicadas y Ambientales UDCA,;Bogotá izenner@udca.edu.co

Estudiante de Ingeniería Agronómica, Universidad de Ciencias Aplicadas y Ambientales UDCA; Bogotá.

Docente Investigador, Facultad de Ingeniería Agronómica, Universidad de Ciencias Aplicadas y Ambientales UDCA,

Bogotá.rmejía@udca.edu.co 


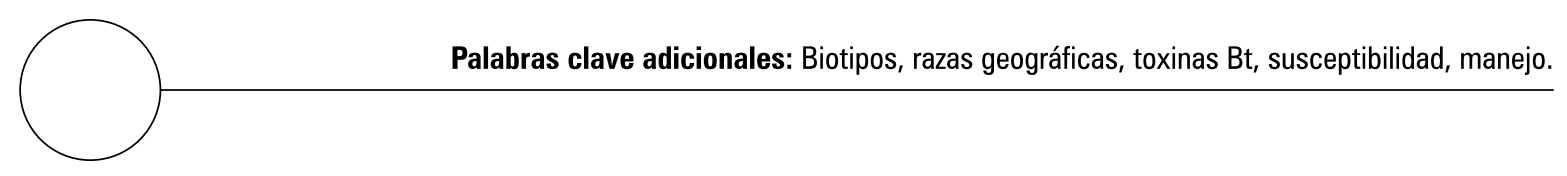

\section{ABSTRACT}

Based on the preliminary experimental data and a critical review of studies executed in North and South America, the possibility of existence of two strains or biotypes of fall armyworm (FAW) Spodoptera frugiperda in Colombia was studied. Moreover, its influence on the management of this polyphagous pest and the prospects of controlling the insect with transgenic corn and cotton hybrids that express one or more Bacillus thuringiensis toxins were analyzed and discussed. Given the fact similarity of $\mathrm{CL}_{50}$ obtained during evaluations for tolerance to Bt toxin Cry1Ac of different FAW strains collected on different hosts and in diverse geographic regions, we assumed the existence of a strain that attacks both corn and cotton and another one that affects rice and pastures. Populations collected on corn and cotton exhibited a tolerance up to 127 times higher than that of pasture population, which further showed a $\mathrm{CL}_{50}$ to the toxin Cry1 $\mathrm{Ab}$ between 3,45 and 5,64 ppm. Likewise, some advances in the attainment of plant species, hosts of the insect genetically transformed with toxins different from those coming from Bt were shown and evaluated.

Additional key words: Host strains, geographic strains, bt toxins, susceptibility, management.
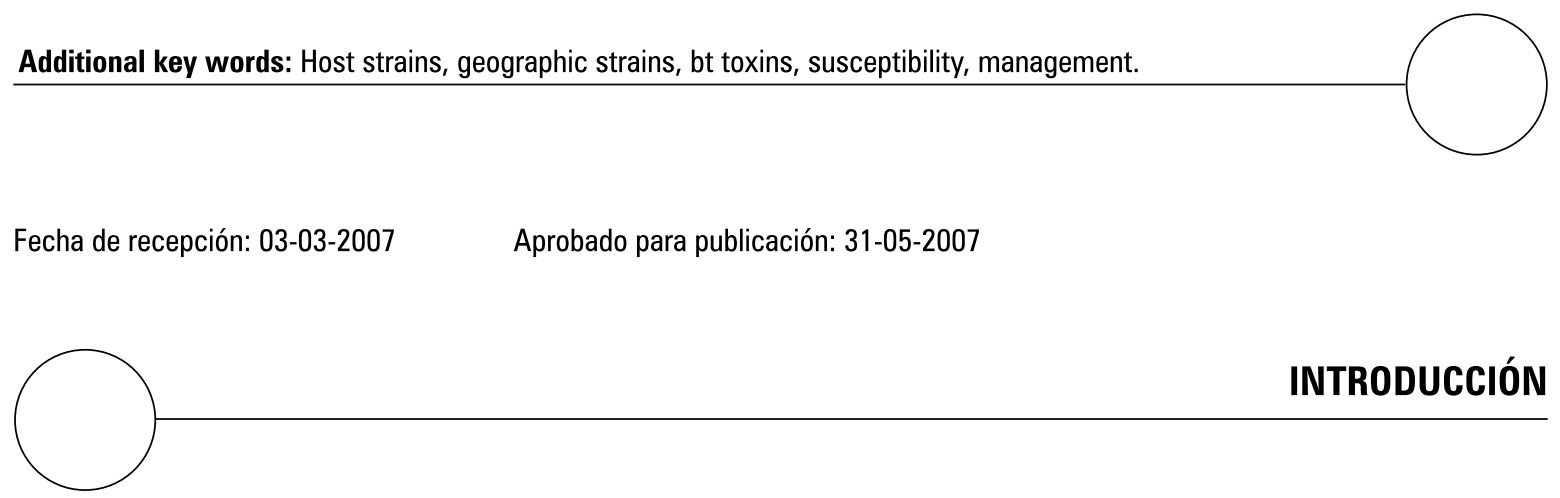

Una de las plagas insectiles de mayor importancia económica en el país es el gusano cogollero del maíz, Spodoptera frugiperda (J.E. Smith) (Lepidoptera: Noctuidae). De acuerdo con Posada (1989), las larvas o gusanos pueden alimentarse de 28 especies vegetales cultivadas, entre las cuales se destacan el maíz, el sorgo, el algodonero, la soya, la higuerilla, el tomate de huerta, la caña de azúcar, el ajonjolí, el arroz, el maní, el melón y el girasol. Prefiere para su alimentación a las gramíneas, cultivadas o no, pero causa pérdidas elevadas a otros cultivos, ante todo, cuando sus poblaciones logran altos niveles durante las épocas de verano y cuando actúan como gusano ejército. No existen estadísticas referentes a pérdidas reales causadas por el insec- to, pero en maíz tecnificado se considera que un $5,6 \%$ de los costos de producción corresponden al control químico de la plaga (URPA, citado por García et al., 1999).

Por esta razón, para su manejo se debe monitorear su presencia en forma cuidadosa, teniendo en cuenta que el insecto se puede pasar de un cultivo a otro y que los pastos y las socas siempre albergan poblaciones peligrosas para el siguiente cultivo.

En gramíneas, tales como maíz y sorgo, la presencia de la plaga se considera endémica, es decir siempre existen poblaciones que causan daño en mayor o menor proporción al cultivo. En las otras plantas el ataque se observa de manera 
impredecible, existen temporadas donde las poblaciones del insecto se consideran de ninguna importancia, mientras que en otras se requieren controles continuos, a menudo inclusive sin éxito por la presencia de altas poblaciones y tolerancia o resistencia del insecto a los productos químicos empleados (Zenner de Polanía y Borrero, 1994; Zenner de Polanía et al., 2006).

El control del insecto ha sido tradicionalmente con productos químicos de diversos ingredientes activos, a veces utilizando también la bacteria comercial Bacillus thuringiensis. En algunas zonas maiceras del país se han realizado tímidos intentos de controles biológicos con liberaciones de parasitoides y con el respeto de la fauna benéfica, representada por coccinélidos, chinches, nitidúlidos y arañas, todos ellos depredadores (Zenner de Polanía \& Saldarriaga, 1986; Ospina, 1999; García et al., 1999).

Si se analiza por ejemplo el comportamiento de mercados de referencia, se observa que los precios de maíz, por lo menos durante los meses de octubre y noviembre de 2006, han mantenido la tendencia positiva, existiendo un marcado incremento en las cotizaciones y una alta demanda del grano (Fenalce, 2006). Se asume que esta tendencia sigue por el incremento en el uso de maíz para la producción de etanol, lo cual tendrá como consecuencia un aumento en el área sembrado en el país, ante todo en la Altillanura, donde el $S$. frugiperda es una plaga endémica y se presenta en altas poblaciones, ante todo durante el primer semestre del año.

De acuerdo a Pashley et al. (1985; 2004) existen dos razas del cogollero del maíz, genéticamente diferentes, la raza de maíz que ocurre en maíz y algodón, y la raza de arroz, asociada con esta planta y el pasto Bermuda (Cynodon dactylon), lo cual podría tener implicaciones en el manejo de la plaga con plantas transgénicas. Es muy probable que tanto en el departamento del Tolima, como en la Altillanura existen las dos razas, cuyo comportamiento frente a los OGM (organismos genéticamente modificados) podría ser diferente. En el Brasil se da por hecho la existencia de los dos biotipos o razas, los cuales están siendo tratadas y manejadas como dos plagas diferentes (Rossato et al., 2006).

En la actualidad las perspectivas del uso de plantas transgénicas con toxinas del $B$. thuringiensis incorporadas, ha despertado mucho interés y controversia en todo el mundo entre los agricultores, los ambientalistas, los ingenieros agrónomos, asistentes técnicos y el público en general. Estas plantas son organismos genéticamente modificadas a las cuales se les introdujo mediante metodologías de ingeniería genética, ADN foráneo de diferente índole. Las plantas a las cuales se ha introducido por ejemplo el gen Cry1Ac del B. thuringiensis var. kurstaki, el cual expresa cristales proteicos delta-endotoxinas, poseen efectividad insecticida contra larvas de algunas especies del Orden Lepidoptera ( $\mathrm{Ta}-$ bashnik et al., 2003; Waquil et al., 2002).

Aunque inicialmente estas plantas transgénicas fueron presentadas por las compañías productoras de semillas como la panacea para disminuir los costos de producción, evitar problemas y pérdidas por determinados insectos, hoy día ya es de conocimiento general que la toxina Cry1Ac no actúa contra todas las plagas lepidópteras de las plantas cultivadas a las cuales ha sido incorporado. Por la adquisición de resistencia de determinadas plagas al Cry1Ac y con el fin de ampliar el espectro de control a otros lepidópteros, actualmente ya se cuenta con la segunda generación de plantas transgénicas, las cuales contienen un coctel de proteínas, además del Cry1Ac, la proteína Cry2Ab (Greenplate et al., 2003). Esta variedad de algodón, denominada Bollgard II, se comercializa en Estados Unidos y Australia, pero todavía no en Colombia.

En el país se dispone al presente del algodonero transgénico NuOpal, tecnología Bollgard®, que proporciona la toxina Cry1Ac. El maíz híbrido transgénico, de diversa procedencia, al cual fue 
introducida la toxina Cry1 $\mathrm{Ab}$, se encuentra bajo los estudios requeridos previa liberación para siembras comerciales en Colombia. Además se esta evaluando maíz transgénico el cual contiene la toxina Cry1F, esta última ya probada y comercialmente disponible en el Brasil (Waquil et al., 2002).

Al evaluar la tolerancia del $S$. frugiperda a los dos Cry mencionados, se observaron diferencias notables en las dosis letales, lo cual motivó ensayos más detallados y revisiones de literatura que podrían facilitar la interpretación de estas diferencias y además mostrar el futuro de las plantas transgénicas resistentes o tolerantes a esta plaga omnívora.

\section{S. FRUGIPERDA Y PERSPECTIVAS DEL USO DE MAÍZ TRANSGÉNICO}

En la tabla 1, se consignan algunos resultados de la determinación de las $\mathrm{CL}_{50} \mathrm{y} \mathrm{CL}_{80}$ para la toxina Cry1Ac obtenidas para $S$. frugiperda procedente de dos regiones del país. Los ensayos, se realizaron bajo condiciones de laboratorio, incluyendo las concentraciones seriadas de 0,001 a 1000 y de 0,002 a 2000 ppm de la toxina incorporada en dieta merídica. Se emplearon por vaso cinco larvas neonatas de la $\mathrm{F}_{1}$ obtenidas en el laboratorio y cada dosis se repitió seis veces. Las observa- ciones de mortalidad larval se realizaron hasta el día siete del inicio del ensayo, día en el cual se tomaron los datos de mortalidad para realizar el análisis Probit.

Analizando estos datos resalta inicialmente la similitud de las concentraciones letales (CL) medias obtenidas para las razas procedentes del Meta y del Tolima, obtenidas de maíz y algodón convencional respectivamente. Este resultado confirma preliminarmente, para Colombia, lo observado por Martinelli et al. (2006) en el Brasil de que las poblaciones de $S$. frugiperda que se desarrollan en maíz y en el algodonero corresponden a la raza de maíz (Pashley et al., 1985), mientras que posibles diferencias genéticas entre estas poblaciones se deben más bien a razas que se desarrollaron en diferentes regiones geográficas. En este contexto sería de gran interés e importancia, desde el punto de vista manejo, poder comparar genéticamente poblaciones del cogollero procedentes de los Valles Interandinos colombianos, de la Costa Atlántica y de la Orinoquía, colectadas en las diversas especies huéspedes de la plaga.

Desde el punto de vista del manejo de la plaga en el algodonero transgénico, se confirma lo observado por Zenner de Polanía et al. (2005) que, la toxina Cry1Ac en las concentraciones disponibles en la planta no ejercen un control satisfactorio del cogollero.

Tabla 1. Respuesta dosis mortalidad para $S$. frugiperda en bioensayos realizados con dieta merídica con dosis seriadas del Cry1Ac incorporado (análisis Probit).

\begin{tabular}{|l|l|r|r|r|r|r|r|}
\hline \multicolumn{1}{|c|}{ Procedencia } & \multicolumn{1}{|c|}{ Cultivo } & \multicolumn{1}{c|}{$\mathrm{CL}_{50}$} & \multicolumn{1}{c|}{$\mathrm{IC} 95 \%$} & \multicolumn{1}{c|}{$\mathrm{CL}_{80}$} & \multicolumn{1}{c|}{$\mathrm{IC} 95 \%$} & Pendiente & $r^{2}$ \\
\hline Pto López & Maíz & 715,3 & $581,1-888,4$ & 1596,0 & $1296,7-1964,3$ & 2,2 & 0,95 \\
\hline $\begin{array}{l}\text { El Espinal } \\
\text { (Fase Migratoria) }\end{array}$ & Pastos & 5,6 & $5,03-6,3$ & 17,6 & $15,7-19,7$ & 2,6 & 0,74 \\
\hline Espinal Algodón & Algodón & 693,7 & $605,8-794,3$ & 1596,0 & $1123,2-1472,7$ & 2,3 & 0,95 \\
\hline El Espinal & Soca Maíz & 191,9 & $174,0-211,6$ & 1440,0 & $1305,8-1587,9$ & 3,4 & 0,92 \\
\hline
\end{tabular}

$\mathrm{IC}=$ Intervalo de confianza.

$r^{2}=$ Coeficiente de determinación. 
La disminución considerable de la CL observada en larvas del cogollero procedentes de soca de maíz (tabla 1), se atribuye a la dilución de la resistencia al Cry1Ac, debido al número de generaciones del insecto transcurrido en el cultivo de maíz y probablemente otro en la soca, lo cual podría sumar un total de cuatro generaciones sin presión de selección por la toxina Bt. La población se desarrolló en el semestre con veda para la siembra del algodonero, así que no hubo migraciones ente cultivos de maíz y algodonero transgénico, ni posibilidad de intercambio de genes que imparten resistencia. Esta ausencia de presión de selección en el segundo semestre del año en el departamento del Tolima, se debería aprovechar con siembras de maíz convencional, para lograr una mayor susceptibilidad del insecto al Cry1Ac y así lograr un manejo más adecuado de la plaga. Sin embargo, resalta la pronunciada pendiente de la línea de regresión $(3,43)$, la cual indica que a medida que el insecto esté nuevamente sujeto a presión de selección, la tolerancia aumentará mucho más rápido que en ocasiones anteriores.

La población evaluada procedente de pastos (tabla 1) muestra una suscpetibilidad de más de 100 veces mayor al Cry1Ac que las poblaciones colectadas en maíz y algodonero. Este hecho se podría interpretar nuevamente como la existencia de la raza "arroz", la cual se desarrolla indistintamente en arroz y pastos (Pashley et al., 2004; Levy et al., 2002; Murúa y Virla, 2004) y tendría en el departamento del Tolima los huéspedes adecuados a su disposición.

Tradicionalmente la diferenciación de biotipos de insectos se realiza a través de estudios de clasificación taxonómica sobre la base del fenotipo. Sin embargo, debido a la influencia del ambiente, esta diferenciación puede costituirse en una medida inexacta de la diversidad genética que éstas representan. El problema puede ser revertido con análisis genéticos que empleen marcadores de ácido desoxirribonucleico (ADN), los cuales pueden ser usados además en la construcción de ma- pas genéticos y en la identificación y aislamiento de genes y/o grupos de estos. Estas técnicas se basan en dos principios que se pueden utilizar individualmente o de forma combinada: la hibridación de ácidos nucléicos y la reacción en cadena de la polimerasa (PCR) (Cornicle, 2002). La primera incluye la técnica de RFLP (Restriction Fragment Length Polymerphism), la cual se ha utilizado para diferenciar poblaciones de $\mathcal{S}$. frugiperda en el suroriente de los Estados Unidos a partir de la actividad genética del citocromo oxidasa (Nagosii et al., 2006); y la técnica de chips de ADN, cuyo uso más importante es el análisis de marcadores polimórficos. Con respecto a los marcadores basados en PCR, se cuenta con las técnicas de polimorfismo del ADN amplificado al azar (RAPD), polimorfismos de la longitud de los fragmentos amplificados (AFLP) y microsatélites. La selección de la técnica depende del objetivo trazado, pero en general todas ofrecen información exacta a diferentes niveles taxonómicos. Sin embargo, es importante considerar que el uso de estas tecnologías es limitado en los países en desarrollo, por el alto costo de los equipos y los reactivos. Sería, por lo tanto, conveniente poder confirmar, si mediante bioensayos sencillos y estudios toxicológicos, se podría determinar, no solamente la tolerancia a toxinas, sino la existencia de biotipos y razas diferenciales geográficas y de huéspedes.El maíz híbrido trangénico bajo evaluación por parte del Instituo Colombiano Agropecuario ICA, posee la toxina Cry1Ab, con la cual se realizaron algunos bioensayos preliminares contra el cogollero del maíz. El Cry1Ab empleado e incorporado a la dieta merídica en dosis seriadas de 0,001 a 100ppm, fue aislado del serotipo de $E$. coli (EC54), donado por el Bacillus Genetic Stock Center (Ohio State University). La población de $S$. frugiperda procedía de El Espinal, fase migratoria (gusano ejército), colectada en potreros, por lo cual nuevamente se asume que se trata de la raza "arroz". Se evaluó en dos ensayos la primera generación de laboratorio, utilizando la misma metodología utilizada para la evaluación del Cry1Ac. 
El análisis Probit del primer bioensayo arrojó una $\mathrm{CL}_{50}$ de 5,64 ppm, con un intervalo de confianza de 5,03-6,32 ppm, $\mathrm{CL}_{80} 17,64 \mathrm{ppm}$, con un intervalo de confianza de 15,74-19,77 ppm, una pendiente de la línea de regresión de 2,631 y un coeficiente de determinación, $\mathrm{R}^{2}$, de 0,74 . Para el segundo ensayo estos datos corresponden a: $\mathrm{CL}_{50}$ de 3,45 ppm, intervalo de confianza de 3,143,79 ppm, $\mathrm{CL}_{80} 7,38 \mathrm{ppm}$, intervalo de confianza de 6,72-8,10 ppm, una pendiente de la línea de regresión de 3,95 y un coeficiente de determinación, $R^{2}$, de 0,71 . De estos resultados llama la atención la pendiente pronunciada, lo cual se puede interpretar como una tendencia alta del aumento del porcentaje de mortalidad, a medida que se aumenta la dosis.

Estas evaluaciones relacionadas con la susceptibilidad al Cry1Ab se deben realizar con poblaciones procedentes de maíz y algodonero, ya que aparentemente la raza "arroz" es mucha más susceptible a los Crys del $B$. thuringiensis y no permitirá extrapolar conclusiones acerca del comportamiento del híbrido de maíz con el Cry1 Ab incorporado. La mayor susceptibilidad de la raza "arroz" sería ventajosa al ser incorporado cualquiera de las dos toxinas a variedades de arroz para el manejo del cogollero. Esta afirmación ya se encuentra confirmada por el estudio realizado por Abreu et al. (2004) en Cuba, quienes afirman que el Cry1Ca y el híbrido Cry1A-Cry1Ca imparten resistencia al arroz contra $S$. frugiperda.

En los Estados Unidos, se logro obtener resultados positivos con el uso de maíz dulce transgénico que contenía la toxina Cry1Ab, en varios experimentos de campo realizados por Lyncii et al. (1999a; 1999b). Concluyeron estos autores que el maíz dulce Bt ofrece una excelente oportunidad para un manejo ambientalmente favorable de este cultivo y además, aseveran que es poco probable que $S$. frugiperda y el gusano de la ma- zorca, Helicoverpa zea, desarrollen resistencia a la variedad, por las altas concentraciones de las toxinas expresadas en este maíz dulce.

Abel y Adamczyk (2004) analizando el desarrollo larval del cogollero en diferentes partes de la hoja de maíz trangénico Cry1Ab, lograron la mortalidad más alta, un $71,5 \%$, en la mitad de la hoja número 9 en desarrollo, en comparación con la mitad de la hoja número 7 y la punta de la misma hoja, que proporcionaron respectivamente un 39,3 y $17,6 \%$ de mortalidad. Concluyen estos autores que para proteger a las plantas de maíz de daños económicamente importantes del $S$. frugiperda, debe amplificarse la concentración del Cr$\mathrm{y} 1 \mathrm{Ab}$ en la planta o realizar investigaciones con el fin de buscar otros métodos de control integrables con el uso del híbrido transgénico. Para Colombia todavía no se han realizado los estudios relacionados con la concentración del cry1Ab que expresa el híbrido de maíz transgénico, del cual el Ministerio de Agricultura ha autorizado la siembra experimental de 35.000 ha para el primer semestre de 2007. La concentración de la toxina que expresa del Cry es dependiente de factores ambientales, tales como la radiación, la temperatura, la intensidad lumínica (Mahon et al., 2002) y los niveles de fertilización (Kleiner et al., 1998), por lo cual, se deberían hacer estudios específicos dependiendo de la región donde se libera el material, para ajustar un plan básico de manejo del cultivo, dirigido a los agricultores.

Para establecer estrategias de manejo de resistencia del cogollero referente a los híbridos de maíz, con la toxina Cry1A(b) o Cry1F, Waquil et al. (2004) en el Brasil estimaron para la primera toxina una mortalidad del $58 \%$ y para la segunda este porcentaje hasta el 100\%. Ya con anterioridad Waquil et al. (2002) habían encontrado un aumento en la tolerancia del insecto al $\operatorname{Cr} 1 \mathrm{~A}(\mathrm{~b})$ después de ejercer presión de selección durante cuatro generaciones de la plaga. 


\section{S. FRUGIPERDA Y EL ALGODÓN TRANSGÉNICO}

Asumiendo que un solo biotipo del cogollero afecta tanto al maíz como al algodonero, vale la pena analizar el comportamiento del insecto en la variedad del algodonero transgénico que se siembra actualmente en el país y en el algodonero piramidal.

Las siembras de algodón transgéncio, Bollgard $₫$, el cual posee la endo-toxina Cry1Ac, se iniciaron en el país en el segundo semestre de 2003 en la zona algodonera de la costa, Córdoba y Sinú. En esta región el gusano cogollero del maíz, aunque se presenta periódicamente como plaga de importancia económica (Álvarez, 1991), no se observó durante esta cosecha en poblaciones elevadas, por lo cual no se registraron fallas en su control. Las siembras en el interior, durante el primer semestre de 2004 mostraron ataques no esperados del cogollero, ya que las evaluaciones previas a la liberación del material transgénico en el país, realizados por el Instituto Colombiano Agropecuario, indicaron que éste es tolerante al ataque del $S$. frugiperda.

La literatura internacional indica que el $S$. frugiperda no es plaga de interés en los países de climas templados, en los cuales se crearon las variedades de algodón transgénico, por lo cual en estas regiones no se realizaron los estudios básicos, recomendados por la EPA (1998), con esta especie insectil. El Bollgard $®$ fue modificado genéticamente para controlar el bellotero del algodonero Heliothis virescens, plaga principal al nivel americano de esta fibra.

Para determinar el efecto que puede ejercer el Cry1Ac incorporado al algodonero en el desarrollo de las larvas del gusano cogollero, el grupo de investigación Fitosanidad de la UDCA inició una serie de experimentos, alimentando las larvas con tejido fresco de la planta, cotiledones, hojas terminales y botones florales, y con dieta artificial, a la cual se incorporó la toxina. El te- jido fresco procedente de cultivos del área de El Espinal (Tolima) se escogió de manera secuencial, teniendo en cuenta que la literatura indica que la concentración de la toxina disminuye a medida que la planta llega a su madurez fisiológica (Greenplate, 1999; Adamczyk y Sumerford, 2001).

Los resultados de estos experimentos indican que las concentraciones del Cry1Ac, en las tres estructuras del algodonero evaluadas no son lo suficientemente altas como para ocasionar la muerte a las larvas del gusano cogollero del maíz a través del tiempo, pero sí ocasionan un incremento en la duración del ciclo de vida del insecto (Zenner de Polanía et al., 2005). La determinación de la concentración del Cry1Ac en cotiledones y hojas de terminales liofilizados, mostró como datos preliminares, que los cotiledones poseen una concentración de $0,9 \mu \mathrm{g} / \mathrm{mg}$ de la toxina, mientras que en las hojas terminales solamente se detectaron $0,3 \mu \mathrm{g} / \mathrm{mg}$ (datos sin publicar). Adamczyk y Sumerford (2001) evaluando la cantidad de Cry1Ac en hojas terminales de 13 variedades transgénicas, encontraron que el nivel de la toxina disminuye constantemente durante la temporada algodonera, iniciándose con una concentración promedia de 1,5 ppm para llegar a solamente 0,5 ppm después de casi dos meses. Esta concentración no proporciona una mortalidad al estado inmaduro del cogollero.

Al incorporar dosis seriadas a la dieta artificial, con la dosis de 1 ppm, se observó al cabo de 26 días solamente un porcentaje de mortalidad de $20,83 \%$, con las dosis de 0,01 y 0,1 ppm se obtuvo un $100 \%$ de supervivencia, mientras que con las dosis altas, 10, 100 y 1000 ppm, la mortalidad de un $100 \%$ solamente se manifestó en el día 28 , de haberse iniciado el experimento, en adelante (Zenner de Polanía et al., 2005).

Para fines prácticos de un manejo integrado del cogollero en el cultivo de algodón transgéncio se destaca la aparente ausencia de un efecto nega- 
tivo del Cry1Ac sobre el parasitoide huevo-larva de S. frugiperda, Chelonus insularis (Hymenoptera: Braconidae). La alimentación de larvas parasitadas de la plaga con tejido fresco del algodonero transgénico, cotiledones y hojas terminales, no tuvo consecuencias para el parasitoide. Un 83,60\% del insecto benéfico alcanzó el estado adulto. Resultados similares fueron encontrados por Zenner de Polanía et al. (2006), al evaluar la influencia del parasitismo sobre la susceptibilidad del cogollero a un producto comercial a base de $B$. thuringiensis. Geng et al. (2006) evaluaron el efecto del polen de algodón transgénico, que expresa el Cry1Ac y el gen CpTI ("cowpea trypsin inhibitor") sobre el parasitoide huevo-huevo Trichogramma chilonis. Encontraron que para los adultos el alimento consistente en polen, procedente tanto de algodón transgénico como algodón convencional, y miel, representa una dieta completa para la reproducción y supervivencia del parasitoide. Teniendo en cuenta que diversas especies de Trichogramma son liberadas para el manejo de plagas lepidópteros, ante todo en el algodonero, en el país, se consideran relevantes estudios sobre el efecto del algodonero transgénico sobre estos controladores biológicos.

A las variedades de algodón, consideradas como de la "segunda generación", se incorporaron dos toxinas Cry1Ac y Cry2Ab con el fin de sobrellevar la tolerancia que mostraron algunas especies de Heliothis. Aparentemente todavía no existen trabajos básicos que analizan la eficacia del Cry2Ab contra $S$. frugiperda, estudios que son prioritarios para poder brindar a los cultivadores de algodón maneras de manejar esta plaga una vez liberada la planta transgénica. Al respecto Stewart et al., ya en el año 2001, mencionan que cultivares de algodón que expresan las dos toxinas del Bt, Cry1Ac y Cry2Ab fueron más tóxicas a algunas plagas, entre las cuales figura $S$. frugiperda, que los cultivares que solamente expresan el Cry1Ac.

S. frugiperda es plaga endémica en el departamento del Tolima, donde se siembra algodón du- rante el primer semestre del año y maíz y sorgo durante el segundo. Además, no se puede descartar que el biotipo de "arroz" del cogollero ataca ocasionalmente al algodonero y al maíz, siendo cultivado el arroz durante ambos semestres. Los maíces transgénicos, probablemente disponibles en el futuro, y los cuales se están evaluando en la actualidad en el país por parte del ICA, poseen incorporado, ya sea el Cry1Ac, el Cry1Ab o el Cry1F. Ya se mencionó la poca eficacia del Cry1Ac contra la plaga y todavía no se conocen los resultados de los estudios con los híbridos de maíz que contienen las otras dos toxinas. Sin embargo, se anticipa la presencia de toxinas Bt similares en el algodonero y el maíz.

Por lo expuesto en este análisis, los estudios básicos y el posterior manejo apropiado de los híbridos transgénicos de maíz Bt y del algodonero piramidal Bt en nuestro medio, jugarán un papel muy relevante en la posibilidad de obtener resultados satisfactorios de control del insecto.

\section{S. FRUGIPERDA Y OTROS CULTIVOS TRANSGÉNICOS}

La existencia de cultivos genéticamente modificados, para fines diferentes al control del cogollero, tales como caña de azúcar, tomate de huerta, arroz y soya, demuestra nuevamente la ausencia o poca motivación de las empresas de los países desarrollados, productoras de plantas transgénicas, en el control del gusano cogollero del maíz. Se confía que en el futuro esta tendencia negativa cambie y se podrá contar también con esta herramienta para los cultivos del trópico y subtrópico.

A continuación y de manera ilustrativa se mencionan algunos ejemplos de estos cultivos transgénicos, útiles para el manejo de otras plagas insectiles, la mayoría no existentes en el país.

Tanto la caña de azúcar como el arroz y el tomate transgénico expresan una toxina, la lectina, derivada de una planta de la familia Amaryllidaceae 
(Galanthus nivalis) (GNA), la cual actúa también contra lepidópteros y consumida por las plagas puede causar ninguna o alta mortalidad, según el comportamiento alimenticio del insecto. GNA afecta por ejemplo de manera negativa al barrenador mexicano del arroz que ataca caña, pero no le causa mortalidad al barrenador de la caña, Diatraea saccharalis (Sétamou et al., 2002).

De posible interés para Colombia podría ser el arroz transgénico (GNA), el cual muestra un elevado nivel de resistencia contra el saltahojas de dorso blanco, Sogatella furcifera y otros insectos chupadores de importancia económica de este cultivo en países como la India y el Japón (Nagadhara et al., 2004; Foissac et al., 2000). Igualmente vale la pena mencionar que a nivel mundial Irán liberó el primer arroz Bt al cual se incorporó el Cry1 Ab contra los barrenadores de tallo (Aguilba, 2005), mientras que científicos cubanos indican que plantas de arroz transgénico que expresan los genes Cry1Ca y el híbrido Cry1A-Cry1Ca son resistentes a $S$. frugiperda (Abreu et al., 2004). Recientemente en la China se incorporó al arroz "japónica" tanto el Cry1Ac como el gen CpTI y se dispone de una serie de arroces transgéncios, los cuales están siendo evaluados contra el barrenador de tallo Chilo suppresalis (Han et al., 2006). Indican estos autores que bajo condiciones de campo estas líneas ofrecen una alta eficacia contra el insecto. Sería interesante poder evaluar en el país líneas de arroz que contienen estos genes por su efecto contra $S$. frugiperda y barrenador del tallo_de la caña de azúcar $D$. saccharalis.

En tomate de huerta, Wakefield et al. (2006) estudiaron el efecto del GNA contra la polilla del tomate, Lacanobia oleracea (Lepidoptera: Noctuidae), bajo condiciones de laboratorio. Encontraron una disminución en la duración del ciclo de vida de la plaga, indicando, en vez de un efecto negativo un efecto positivo en el desarrollo del insecto. Todo lo contrario fue hallado por Gatehouse et al. (1997), quienes obtuvieron una reducción en la biomasa del insecto de un $48 \%$, al alimentar las larvas con tejido vegetal GNA bajo condiciones de invernadero. Teniendo en cuenta que el cogollero del maíz pertenece también a la familia Noctuidae, podría ser de interés para Colombia investigar su comportamiento frente al GNA.

La soya transgénica resistente al herbicida glifosato podría presentar resistencia a algunos insectos plagas, por los cual Morjan y Pedigo (2002) evaluaron su efecto sobre el lepidóptero de la familia Noctuidae, Hypena scabra (F.). No encontraron diferencias entre el desarrollo del insecto en comparación con variedades de soya no transgéncias. Es probable que en el futuro se incorpore también a la soya algún Cry del Bt, lo cual sería de interés para Colombia para aumentar de pronto la productividad del cultivo y hacerlo competitivo a nivel, por lo menos, suramericano.

De lo expuesto en este texto se concluye que las entidades competentes y responsables de la evaluación de cultivos transgénicos en el país, tienen por delante una enorme tarea de investigación básica y aplicada, lo mismo que la búsqueda de genes nativos que podrían ser útiles en el manejo de insectos plagas, al ser incorporado a cultivos de importancia en Colombia. 


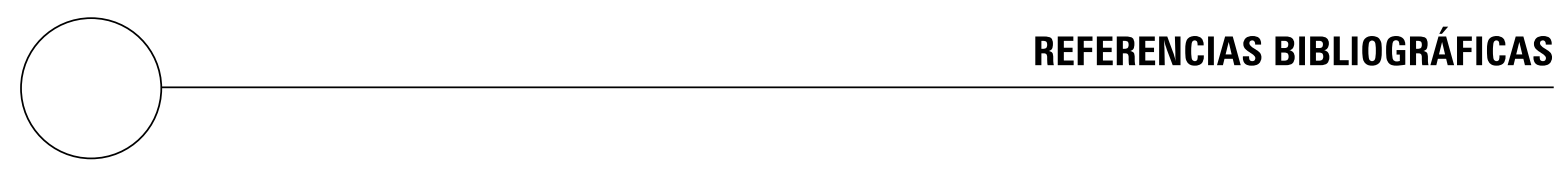

Abel, C.A. y J.J. Adamzcyk, Jr. 2004. Relative concentration of $\mathrm{Cr} 1 \mathrm{~A}$ in maize leaves and cotton bolls with diverse chlorophyll content and corresponding larval development of fall armyworm /Lepidoptera: Noctuidae) and southwestern corn borer (Lepidoptera: Pyralidae) on maize whorl leaf profiles. J. Econ. Entomol. 97(5), 1737-1744.

Abreu, D.; A. Sánchez; M. Pérez; O. Valdivia; A. González; Y. Coll; C. Hernández; C. Ayra; J.A. Rubi; M. Pujol y R. Armas. 2004. Plantas de arroz transgénicos que expresan los genes modificados cry1Ca y el híbrido cry1A Cry1Ca son resistentes a Spodoptera frugiperda. En: www.redbio.org/rdominicana/redbio2004rd/Memoria_REDBIO_2004/Talleres-PDF/ t15-PDF/t15-02pdf; consulta: marzo 2006.

Adamczyk, Jr., J.J. y D.V. Sumerford. 2001. Potential factors impacting season-long expression of Cry1Ac in 13 commercial varieties of Bollgard ${ }^{\circledR}$ cotton. J. Insect Sci. 113. En: http://insectscience.org/1.13; consulta: enero 2004.

Aguilba, M. 2005. Iran releases world's first BT rice. AgBioView \& Manilla Bulletin. En: http://www. truthabouttrade.ord/article.asp? $\mathrm{id}=4891$; consulta: marzo2006).

Álvarez, A. 1991. Reseña histórica y aspectos bioecológicos del gusano cogollero del maíz Spodoptera frugiperda (J. E. Smith). En: Memorias Seminario Spodoptera frugiperda (el gusano cogollero) en sorgo, maíz y otros cultivos. Socolen-Cis. Cali, 1314 Junio, 1991. pp.12-16.

Cornicle, T. 2002. Marcadores moleculares, nuevos horizontes en la genética y la selección de plantas. Ed. Felix Varela, La Habana, Cuba. 366 p.

EPA U.S. Environmental Protection Agency. 1998. The environmental protection agency's white paper on Bt plant - pesticide resistance mangement. Office of Pesticide Programs, Washington, D.C. No. 739-S-98001. pp. 1-78.

FENALCE. 2006. Conjuntura Cerealista. No.9. Noviembre-diciembre. Departamento Económico, Bogotá. $2 \mathrm{p}$.

Foissac, X.; N. Thiloc; P. Christou; A.M. Gatehouse y J.A. Gatehouse. 2000. Resistance to green leafhopper (Nephotettix virescens) and brown planthopper (Nilaparvata lugens) in transgenic rice expressing snowdrop lectin (Galanthus nivalis agglutinin; GNA). J. Insect Physiol. 46(4), 573-583.

García, F.; A.T. Mosquera; C.A. Vargas S. y A.L. Rojas.
1999. Manejo integrado del gusano cogollero del maíz Spodoptera frugiperda (J.E.Smith). CORPOICA, Palmira, Boletín Técnico No. 7. 18 p.

Gatehouse, J.A.; A.M. Gatehouse y E. Fitches. 1997. Effects of snowdrop lectin (GNA) delivered vía artificial diet and transgenic plants on the development of tomato moth (Lacanobia oleracea) larvae in laboratory and glasshouse trials. J. Insect Physiol. 43(8), 727-739.

Geng, J.-H.; Z.-R. Shen; K. Song y L. Zheng. 2006. Effect of pollen of regular cotton and transgenic $B t+\mathrm{CPTI}$ cotton on the survival and reproduction of the parasotoid wasp Trichogramma chilonis (Hymenoptera: Trichogrammatidae) in the laboatory. Environm. Entomol. 35(6), 1661-1668.

Greenplate, J.T. 1999. Quantification of Bacillus thuringiensis insect control protein Cry1Ac over time in bollgard cotton fruit and terminals. J. Econ. Entomol. 92(6), 1377-1383.

Greenplate, J.T.; J.W. Mullins; S.R. Penn; A. Dahm; B.J. Reich; J.A. Osborn; P.R. Rahn; L. Ruschke y Z.W. Shappley. 2003. Partial characterization of cotton plants expressing two toxin proteins from Bacillus thuringiensis: relative toxin contribution, toxin interaction, and resistance management. J. Appl. Entomol.127, 340-347.

Han, L.-Z.; K-M. Wu; Y-F. Peng; F. Wang y Y.-Y. Guo. 2006. Evaluation of transgenic rice expressing Cry1Ac and CpTI against Chilo suppressalis and intrapopulation variation in susceptibility to Cr1Ac. Environm. Entomol. 35(5), 1453-1559.

Kleiner, K.; K. Raffa; D. Elli y H. Brent. 1998. Effect of nitrogen availability on the growth and phytochemistry of hibrid poplar and the efficacy of the Bacillus thuringiensis cry1A(a) d-endotoxin on gypsy moth. Rev. Can. Rech. 28(7), 1055-1067.

Levy, H.G.; A. García-Maruniak; J.E. Maruniak. 2002. Strain identification of Spodoptera frugiperda (Lepidoptera: Noctuidae) insects and cell line: PCRRFLP of cytochrome oxidase C subunit I gene. Florida Entomol. 85(1), 186-190.

Lyncii, R.E.; B.R. Wiseman; D. Plaisted y D. Warnick. 1999a. Evaluation of transgenic sweet corn hybrids expressing Cry1A(b) toxin for resitance to corn earworm and fall armyworm (Lepidoptera: Noctuidae). J. Econ. Entomol. 92(1), 246-252.

Lyncii, R.E.; B.R. Wiseman; D. Plaisted y D. Warnick. 1999b. Management of corn earworm and fall ar- 
myworm (Lepidoptera: Noctuidae) injury on a sweet corn hybrid expressing a cry $1 \mathrm{~A}(\mathrm{~b})$ gene. J. Econ. Entomol. 92(5), 1217-1222.

Mahon, R.; J. Finnegan; K. Olsen y L. Lawrence. 2002. Environmental stress and the efficacy of Bt cotton. The Austr. Cottongrower. March-April, 18-21.

Martinelli, S.; R. Montrazi Barata; M.I. Zucchi; M. de Castro Silva-Filiio y C. Omoto. 2006. Molecular variability of Spodoptera frugiperda (Lepidoptera: Noctuidae) populations associated to maize and cotton crops in Brazil. J. Econ. Entomol. 99(2), 519-526.

Morjan, W.E. y L.P. Pedigo. 2002. Suitability of transgenic glyphosate-resistant soybeans to green cloverworm (Lepidoptera: Noctuidae). J. Econ. Entomol. 95(6), $1275-1280$

Murúa, G. y E. Virela. 2004. Population parameters of Spodoptera frugiperda (Smith) (Lep.; Noctuidae) fed on corn and two predominant grasses in Tucuman (Argentina). Acta Zoolo. Mex. 20(1), 199-210.

Nagadhara, D.; S. Ramesh; I.C. Pasalu; Y.K Rao; N.P. Sarma; V.D. Reddy y K.V. Rao. 2004. Transgenic rice plants expressing the snowdrop lectin gene (gna) exhibit high level resistance to the whitebacked planthopper (Sogatella furcifera). Theor. Appl. Genetics 109(7), 1399-1405.

Nagosii, R.; R. Meagiier; J. Adamczyk; K. Braman; R. Brandenburg; G. Nuessly. 2006. New restriction fragment length polymorphism in the cytochrome oxidase 1 gene faciltates host strain identification of fall armyworm (Lepidoptera: Noctuidae) population in the southeastern United states. J. Econ. Entomol. 99(3), 671-677.

Ospina, J.G. 1999. Tecnología del cultivo de maíz. Fondo Nacional Cerealista, Fenalce. Produmedios, Bogotá. pp. 128-138.

Pashley, D.P.; S.J. Johnson y A.N. Sparks. 1985. Genetic population structura of migratory moths: the fall armyworm (Lepidoptera: Noctuidae). Ann. Entomol. Soc. Am. 78, 756-762.

Pashley, D.P.; M. McMichael y J.F. Silvain. 2004. Multilocus genetic analysis of host use, introgression, and speciation in host strains of fall armyworm (Lepidoptera: Noctuidae). Ann. Entomol. Soc. Am. 97(5), 1034-1044.

Posada, L.1989. Lista de insectos dañinos y otras plagas en Colombia. Instituto Colombiano Agropecuario, ICA. Boletín Técnico No. 43. Produmedios, Bogotá. $662 \mathrm{p}$.

Rossato B.; G., M. Silveira G.; A.E. Loeck; M. Zart; A. Medeiros N.; O. Bernardi y F. da Silva A. 2006. Adequação de uma dieta artificial para os biotipos "milho" e "arroz" de Spodoptera frugiperda (Lepidoptera: Noctuidae). Bragantia 65(2), 317-323.

Sétamou, M.; J.S. Bernal; J.C. Legaspi; T.E. Mirkov y B.C. Legaspi, Jr. 2002. Evaluation of lectin-expressing transgenic sugarcane against stalkborers (Lepidoptera: Pyralidae): effects on life history parameters. J. Econ. Entomol. 95(2), 469-477.

Stewart, S.D.; J.J. Adamczyk, Jr.; K.S. Knighten y F.M. Davis. 2001. Impact of Bt cottons expressing one or two insecticidal proteins of Bacillus thuringiensis on growth and survival of noctuid (Lepidoptera) larvae. J. Econ. Entomol. 94(3), 752-760.

Tabashnik, B.E.; Y. Carriere; T.J. Dennchy; S. Morin; M.S. Sisterson; R.T. Roush; A.M. Shelton y J.Z. Zhao. 2003. Insect resistance to transgenic Bt crops: lessons from the laboratory and fields. J. Econ. Entomol. 96(4), 1031-1038.

Wakefield, M.E.; H.A. Bell; E.C. Fitches; J.P. Edwards y A.M Gatehouse. 2006. Effects of Galanthus nivalis agglutinin (GNA) expressed in tomato leaves on larvae of the tomato moth Lacanobia oleracea (Lepidoptera: Noctuidae) and the effect of GNA on the development of the endoparasitoid Meteorus gyrator (Hymenoptera. Braconidae). Bull. Entomol. Res. 96(1), 43-52.

Waquil, J.M.; F.M.F. Villela; B.D. Siegfried y J.E. Foster. 2004. Actividade biológica das toxinas do Bt, Cry1A(b) e Cry1F em Spodoptera frujiperda (Smith) (Lepidoptera: Noctuidae). Rev. Brasileira de Milho e Sorgo 3(2), 153-163.

Waquil, J.M.; F.M.F. Villela y J.E. Foster. 2002. Resistencia do milho (Zea mays L.) transgenico a lagarta-docartucho, Spodoptera frugiperda (Smith) (Lepidoptera: Noctuidae). Rev. Brasileira de Milho e Sorgo 1(3), 1-11.

Zenner de Polanía, I.; A. Álvarez y S. Barreto. 2006. Influence of parasitism by Chelonus insularis Cresson (Hymenoptera: Braconidae) on the suscpetibility of Spodoptera frugiperda (J.E. Smith) (Lepidoptera: Noctuidae) to insecticides. Neotrop. Entomol. (Brasil). 35(6), 818-822.

Zenner de Polanía, I.; J.A. Álvarez; R. Mejía C. y M.A. Bayona. 2005. Influencia de la toxina Cry1Ac del Bacillus thuringiensis sobre el desarrollo del cogollero del maíz, Spodoptera frugiperda (J.E. Smith). Rev. U.D.C.A Actualidad \& Divulgación Científica 8(2), 129-139.

Zenner de Polanía, I. y F. Borrero. 1994. Resistencia del cogollero del maíz, Spodoptera frugipeda (J.E. Smith) a algunos insecticidas y su manejo. En: Memorias Seminario Internacional sobre el cultivo de maíz y sorgo sus principales plagas y enfermedades. Comité interinstitucional sobre los cultivos de sorgo y maíz. C.I. Tibaitatá. Enero 25-30, 1993. Produmedios, Bogotá. pp. 35-41.

Zenner de Polanía, I. y A. Saldarriaga V. 1986. Guía para el control de plagas. Tercera revisión. Sección de Producción de Medios. Instituto Colombiano Agropecuario ICA, Tibaitatá. pp. 215-217. 